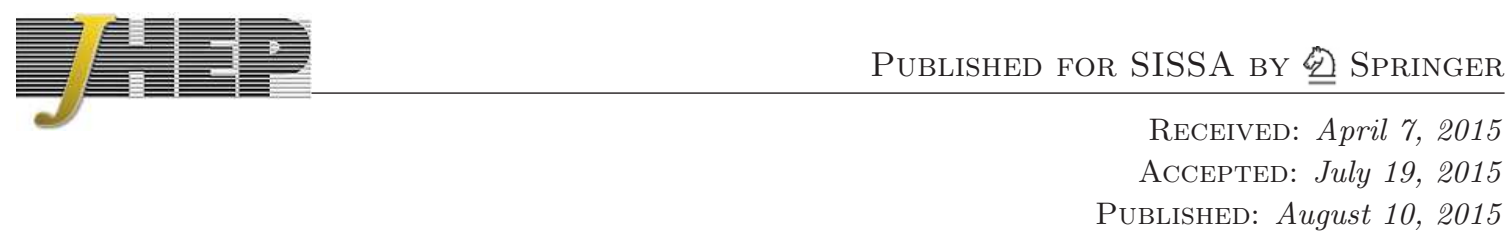

\title{
Superalgebras, constraints and partition functions
}

\author{
Martin Cederwall ${ }^{a}$ and Jakob Palmkvist ${ }^{b}$ \\ ${ }^{a}$ Department of Fundamental Physics, Chalmers University of Technology, \\ SE-412 96 Göteborg, Sweden \\ ${ }^{b}$ Mitchell Institute for Fundamental Physics and Astronomy, Texas A $\mathscr{B} M$ University, \\ College Station, TX 77843, U.S.A. \\ E-mail: martin.cederwall@chalmers.se, jakobpalmkvist@tamu.edu
}

ABSTRACT: We consider Borcherds superalgebras obtained from semisimple finite-dimensional Lie algebras by adding an odd null root to the simple roots. The additional Serre relations can be expressed in a covariant way. The spectrum of generators at positive levels are associated to partition functions for a certain set of constrained bosonic variables, the constraints on which are complementary to the Serre relations in the symmetric product. We give some examples, focusing on superalgebras related to pure spinors, exceptional geometry and tensor hierarchies, of how construction of the content of the algebra at arbitrary levels is simplified.

KEYWORDS: BRST Quantization, BRST Symmetry

ARXIV EPRINT: 1503.06215 


\section{Contents}

1 Introduction 1

2 The superalgebras 3

3 Partition functions 5

$\begin{array}{lll}4 & \text { BRST operator and coalgebra } & 7\end{array}$

$\begin{array}{lll}5 & \text { A proof from denominator formulas } & 11\end{array}$

$\begin{array}{lll}6 & \text { Why is the method not applicable to Lie algebras? } & 14\end{array}$

7 Examples $\quad 16$

$\begin{array}{lll}7.1 & \text { The extreme cases } & 16\end{array}$

$\begin{array}{ll}7.2 D=8 \text { pure spinors and null vectors } & 16\end{array}$

$\begin{array}{lll}7.3 D=10 \text { pure spinors and supergravity forms } & 17\end{array}$

$\begin{array}{lll}7.4 & \text { Superalgebras and Lie algebras } & 19\end{array}$

\section{Introduction}

It is often useful in physics to describe a spectrum of states that appear at various integer levels by means of an associated partition function, especially if the spectrum is infinite. If the states at each level transform in a representation of a Lie algebra, the spectrum of representations may also be obtained from an extended (possibly infinite-dimensional) algebra by a level decomposition. In the present paper we will relate these two approaches to each other, and also to a third important tool in physics: the BRST treatment of reducible constraints.

Our main example is the spectrum of dynamical forms in $D$-dimensional maximal supergravity, which transform in representations of the U-duality group ${ }^{1} E_{n}$, where $n=$ $11-D$. Remarkably, these representations form a Lie superalgebra, which can be extended to an infinite-dimensional Borcherds superalgebra [1-3]. Decomposing it with respect to the $E_{n}$ subalgebra gives back the spectrum of dynamical forms at the positive levels, and also precisely the additional non-dynamical forms allowed by supersymmetry, first determined for $D=10$ in refs. [4-6]. The consistency with supersymmetry was shown in refs. [7-10] using a superspace formulation, generalising bosonic forms to superforms with arbitrary high degrees. However, already in the restriction to the bosonic sector, the wedge product

\footnotetext{
${ }^{1}$ Throughout the paper, we use the notation $E_{n}$ for the split real form, and also for the corresponding Lie algebra. All arguments are however equally valid for the complex Lie algebras.
} 
in the differential algebra, with even and odd forms, naturally gives rise to a superalgebra structure. Up to an arbitrary level $p$ the representations can be also obtained from a level decomposition of the Kac-Moody algebra $E_{n+p}[3,10-12]$. This generalises results for $E_{11}$, which as a special case contains the form spectrum up to $p=D$ [13-15]. However, $E_{11}$ is not enough to accommodate forms with higher degrees, and a rendition of all the representations coming from the Borcherds superalgebra would require a consideration of the infinite-rank algebra $E_{\infty}$.

With a few exceptions, the level decomposition of the Borcherds superalgebra furthermore agrees with the tensor hierarchy of form potentials, field strengths and gauge parameters that arises in the embedding tensor approach to gauged supergravity [16-19]. The tensor hierarchy can be continued to infinity, but misses some of the representations coming from the Borcherds superalgebra. Perfect agreement is instead given by a tensor hierarchy algebra, where the embedding tensor is interpreted as an element at level minus one [20]. Using this algebra all the Bianchi identities and gauge transformations for the gauged theory can be derived in a simple way $[10,21]$. This demonstrates the efficiency of organising representations into a level decomposition of a Lie (super)algebra.

Yet another context where the same infinite sequence of representations appears, and where it cannot be truncated, is exceptional geometry. The exceptional (generalised) diffeomorphisms have infinite reducibility, and the sequence arises as the tower of ghosts for ghosts, describing this reducibility [22]. The connection to partition functions of constrained objects, of which pure spinors [23] is one example, was conjectured already in ref. [22], and used there to correctly regularise the infinite sums arising when counting the degrees of freedom. The same representations occur for tensor fields in exceptional geometry [24], and in the tensor hierarchies considered in [25-28]. The somewhat heuristic approach of ref. [22] provided one of the motivations for the present investigation, which puts the correspondence between the algebra and the constrained objects on a firmer footing.

For $3 \leq D \leq 8$ the U-duality algebra $E_{n}$ is extended to the infinite-dimensional Borcherds superalgebra $\mathscr{B}$ by adding an odd null root $\beta_{0}$ to the simple roots of $E_{n}$. This is the special case that we focus on in this paper, with $E_{n}$ generalised to any semisimple finitedimensional Lie algebra $\mathfrak{g}$. The inner products of $\beta_{0}$ with the simple roots of $\mathfrak{g}$ are assumed to be such that the Serre relations of the Borcherds superalgebra are at most quadratic in the odd Chevalley generators $e_{0}, f_{0}$ corresponding to $\beta_{0}$. Denoting the representation at level $p$ by $R_{p}$, the Serre relations quadratic in $e_{0}$ (say) thus belong to a representation of $\mathfrak{g}$ contained in the symmetric tensor product of $R_{1}$ with itself, with $R_{2}$ as its complement. ${ }^{2}$ It generates an ideal of the free Lie superalgebra generated by $R_{1}$, and at each level $p \geq 2$, the representation $R_{p}$ is the complement that is left when this ideal is factored out. A recursive study of the ideal thus gives all information about the representations $R_{p}$ at any level $p$. In this paper, we will show that the representations $R_{p}$ alternatively, and often more directly, can be determined from the partition function for a bosonic object $\lambda$ in $R_{1}$, subject to the constraint $\left.\lambda^{2}\right|_{R_{2}}=0$. As our main result, we will show that this partition function is the inverse of the partition function for the universal enveloping algebra of $\mathscr{B}_{+}$, the subalgebra of $\mathscr{B}$ at positive levels.

\footnotetext{
${ }^{2}$ With the complement of a representation $R$ in another $R^{\prime}$ we mean the quotient $R^{\prime}-R$.
} 
The paper is organised as follows. In section 2 we describe in more detail the Lie superalgebras that we consider, and how they are constructed from the Chevalley generators and the Serre relations. In section 3 we introduce the partition functions that we use in section 4 to state our results and give them an interpretation in terms of a BRST operator. The argument of section 4 corresponds roughly to the heuristic argument of ref. [22]. We then prove the result in section 5 using the denominator formula for Borcherds superalgebras. Section 6 addresses the question why the method is not applicable to Lie algebras (extensions by an additional even real root). In section 7 we present and discuss some examples.

\section{The superalgebras}

Let $\mathfrak{g}$ be a semisimple finite-dimensional Lie algebra of rank $r$ with simple roots $\alpha_{i}(i=$ $1, \ldots, r)$. We recall that they form a basis of a euclidean space, and from their mutual inner products we get the Cartan matrix $a_{i j}$ of $\mathfrak{g}$ by

$$
a_{i j}=\left(\alpha_{j}, \alpha_{i}^{\vee}\right)=2 \frac{\left(\alpha_{j}, \alpha_{i}\right)}{\left(\alpha_{i}, \alpha_{i}\right)},
$$

where $\alpha_{i}^{\vee}=2 \alpha_{i} /\left(\alpha_{i}, \alpha_{i}\right)$ is the coroot of $\alpha_{i}$.

The construction of a Lie algebra from a basis of simple roots can be generalised to inner product spaces which are not necessarily euclidean, and even from Lie algebras to Lie superalgebras. Semisimple finite-dimensional Lie algebras are then generalised to KacMoody (super)algebras, which in turn are generalised further to Borcherds (super)algebras. Thus Borcherds superalgebras is a very general concept, but in this paper we only consider the special cases described below, motivated by their simplicity and by their appearance in the examples that we will study in section 7 . We refer the reader to refs. [29-32] for more general definitions and other details about Borcherds and Kac-Moody (super)algebras.

The Borcherds superalgebras that we consider are infinite-dimensional superextensions of semisimple finite-dimensional Lie algebras, obtained by adding an odd null root to the simple roots. Let $\mathscr{B}$ be such an extension of $\mathfrak{g}$, with simple roots $\beta_{I}(I=0,1, \ldots, r)$. Thus $\beta_{0}$ is odd and null, $\left(\beta_{0}, \beta_{0}\right)=0$, whereas $\beta_{i}=\alpha_{i}$ are even and real, $\left(\beta_{i}, \beta_{i}\right)>0$. The Cartan matrix $B_{I J}$ of $\mathscr{B}$ is obtained from $a_{i j}$ by adding an extra column

$$
B_{i 0}=\left(\beta_{0}, \beta_{i}^{\vee}\right)=2 \frac{\left(\beta_{0}, \beta_{i}\right)}{\left(\beta_{i}, \beta_{i}\right)}
$$

and an extra row $B_{0 I}=\left(\beta_{I}, \beta_{0}\right)$, including the diagonal entry $B_{00}=\left(\beta_{0}, \beta_{0}\right)=0$. The additional off-diagonal entries $B_{i 0}$ are required to be non-positive integers, like $B_{i j}=a_{i j}$ for $i \neq j$. We assume furthermore that $B_{I J}$ is non-degenerate, and for each $i=1, \ldots, r$, either $B_{0 i}=0$ or $B_{0 i}=-1$.

Adding an extra column and row to the Cartan matrix of $\mathfrak{g}$ corresponds to adding an extra node to the Dynkin diagram of $\mathfrak{g}$, connected with $\left|B_{i 0}\right|$ lines to node $i$. Following ref. [29] we indicate that $\beta_{0}$ is both null and odd by painting the corresponding node "grey" 
(which means that it looks like $\otimes$ ), and let the other nodes, representing real even roots, be white. For example, the Dynkin diagram

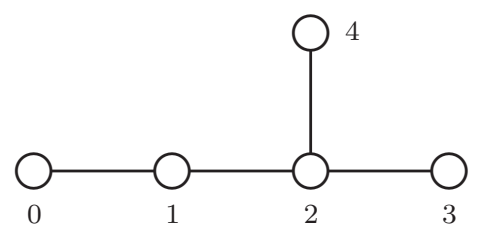

corresponds to the Cartan matrix

$$
B_{I J}=\left(\begin{array}{ccccc}
0 & -1 & 0 & 0 & 0 \\
-1 & 2 & -1 & 0 & 0 \\
0 & -1 & 2 & -1 & -1 \\
0 & 0 & -1 & 2 & 0 \\
0 & 0 & -1 & 0 & 2
\end{array}\right)
$$

We will come back to this algebra, among other examples, in section 7 .

To each simple root $\beta_{I}$ of $\mathscr{B}$ we associate Chevalley generators $e_{I}, f_{I}$ and $h_{I}$, and $\mathscr{B}$ is then defined as the Lie superalgebra generated by these elements (of which $e_{0}$ and $f_{0}$ are odd and the others even) modulo the Chevalley relations

$$
\left[h_{I}, e_{J}\right]=B_{I J} e_{J}, \quad\left[h_{I}, f_{J}\right]=-B_{I J} f_{J}, \quad\left[e_{I}, f_{J}\right\}=\delta_{I J} h_{J},
$$

and the Serre relations

$$
\left(\operatorname{ad} e_{I}\right)^{1-B_{I J}}\left(e_{J}\right)=\left(\operatorname{ad} f_{I}\right)^{1-B_{I J}}\left(f_{J}\right)=0
$$

for $I \neq J$. For $I=0$ the Serre relations (2.5) can equivalently be replaced by

$$
\left\{e_{0}, e_{0}\right\}=\left\{f_{0}, f_{0}\right\}=0
$$

since, by the Jacobi identity,

$$
\frac{1}{2}\left[\left\{e_{0}, e_{0}\right\}, e_{J}\right]=\left\{e_{0},\left[e_{0}, e_{J}\right]\right\}, \quad \frac{1}{2}\left[\left\{f_{0}, f_{0}\right\}, f_{J}\right]=\left\{f_{0},\left[f_{0}, f_{J}\right]\right\},
$$

which gives

$$
\left[\left\{e_{0},\left[e_{0}, e_{J}\right]\right\}, f_{J}\right]=\left\{e_{0}, e_{0}\right\}, \quad\left[\left\{f_{0},\left[f_{0}, f_{J}\right]\right\}, e_{J}\right]=\left\{f_{0}, f_{0}\right\}
$$

if $B_{J 0}=-1$. Thus in this case the ideal generated by $(2.5)$ is contained in the ideal generated by (2.6), and conversely. If $B_{J 0}=0$, there is already a redundance in (2.5) because of the antisymmetry of the bracket, so replacing (2.5) by (2.6) in this case simply amounts to removing one of two equivalent relations in (2.5).

For any integer $p$, let $\mathscr{B}_{p}$ be the subspace of $\mathscr{B}$ spanned by all root vectors corresponding to roots $\beta=p \beta_{0}+\alpha$, where $\alpha$ is a linear combination of the real simple roots $\beta_{i}=\alpha_{i}$, and, if $p=0$, by the Cartan elements $h_{I}$. Since $\mathscr{B}$ is the direct sum of all these subspaces, and $\left[\mathscr{B}_{p}, \mathscr{B}_{q}\right\} \subseteq \mathscr{B}_{p+q}$, this decomposition is a $\mathbb{Z}$-grading of $\mathscr{B}$, leading to a 
level decomposition of its adjoint representation under the subalgebra $\mathfrak{g} \subset \mathscr{B}_{0}$, with $\mathscr{B}_{p}$ consisting of a (maybe reducible) representation ${ }^{3}$ of $\mathfrak{g}$ at level $p$. We will throughout the paper denote this representation $R_{p}$.

Let $\mathscr{B}_{+}$and $\mathscr{B}_{-}$be the subalgebras of $\mathscr{B}$ spanned by elements at positive and negative levels, respectively, and let $\tilde{\mathscr{B}}_{ \pm}$be the free Lie superalgebra generated by $\mathscr{B}_{ \pm 1}$. The Serre relations (2.6) generate an ideal of $\tilde{\mathscr{B}}$ which is the direct sum of two subalgebras $\mathscr{D}_{ \pm}$, where $\mathscr{D}_{ \pm} \subset \tilde{\mathscr{B}}_{ \pm}$(and is the maximal such ideal). The Borcherds superalgebra $\mathscr{B}$ is then obtained by factoring out this ideal from $\tilde{\mathscr{B}}$, and in particular $\mathscr{B}_{+}$is obtained by factoring out $\mathscr{D}_{+}$ from $\tilde{\mathscr{B}}_{+}$, the free Lie superalgebra generated by $\mathscr{B}_{1}$. The ideal $\mathscr{D}_{+}$of $\tilde{\mathscr{B}}$ is generated by the element $\left\{e_{0}, e_{0}\right\}$ at level two, which is set to zero in one of the Serre relations (2.6). However, considered as an ideal of $\tilde{\mathscr{B}}_{+}$only, it is generated by all elements at level two in $\mathscr{D}_{+}$, which are not only $\left\{e_{0}, e_{0}\right\}$ but also those obtained from $\left\{e_{0}, e_{0}\right\}$ by successively acting with $\mathfrak{g}$. These elements form a representation $R_{2}{ }^{\perp}$, which is the complement of $R_{2}$ in $\vee^{2} R_{1}$ (the symmetrised tensor product of $R_{1}$ with itself) with a lowest weight vector $\left\{e_{0}, e_{0}\right\}$. It then follows from the Chevalley relations that the Dynkin labels of the lowest weight of $R_{2} \perp$ are given by $\lambda_{i}=2 B_{i 0}$.

Using a basis $E_{M}$ of $\mathscr{B}_{1}$ (so that the index $M$ corresponds to the representation $R_{1}$ ), we can summarize the above construction of $\mathscr{B}_{+}$by saying that it is the Lie superalgebra generated by the odd elements $E_{M}$ modulo the "covariant Serre relations"

$$
\left.\left\{E_{M}, E_{N}\right\}\right|_{R_{2} \perp}=0 \text {. }
$$

Recursive use of these relations (and of course of the Jacobi identity) gives complete information about the representation $R_{p}$ at arbitrarily high levels $p$. In section 4 we will describe how this information can be efficiently encoded into partition functions, which will be discussed next.

\section{Partition functions}

The purpose of this section is to introduce and define notation for the partition functions we use to state our results.

The partition functions we will consider count the number of bosonic and fermionic objects occurring with some $\mathbb{Z}$-weight, or level, and some additional quantum numbers. In an "unrefined" partition function, only counting the number of states per level, the presence of some set of $N$ linearly independent objects with weight $p$ corresponds to a term $\sigma^{p} N t^{p}$, where $\sigma=1$ for bosons and $\sigma=-1$ for fermions. This means we are really considering partition functions twisted by fermion number, which of course has the advantage that the partition function for a fermionic variable, or "creation operator",

$$
Z^{F}(t)=1-t
$$

and that of a bosonic one,

$$
Z^{B}(t)=(1-t)^{-1}
$$

are each other's inverses.

\footnotetext{
${ }^{3}$ Following the physics terminology, we use the term "representation" also for the module of the representation, i.e., the vector space it acts on.
} 
More refined partition functions may be defined if additional quantum numbers are available. In a typical case, a variable or operator will transform in some representation $R$ of a Lie algebra $\mathfrak{g}$. A refined partition function encodes completely the representations of all states, and is a formal power series in a variable $t$ (corresponding to the grading) with coefficients in the unit ring of $\mathfrak{g}$-representations (under tensor product).

The basic examples are the refined partition functions for fermionic and bosonic creation operators in $R$ :

$$
\begin{aligned}
& \mathscr{Z}_{R}^{F}(t)=\bigoplus_{p=0}^{|R|}(-t)^{p} \wedge^{p} R, \\
& \mathscr{Z}_{R}^{B}(t)=\bigoplus_{p=0}^{\infty} t^{p} \vee^{p} R .
\end{aligned}
$$

Here, we use $\wedge$ and $\vee$ for antisymmetric and and symmetric products, respectively, and $|R|$ denotes the dimension of a representation $R$. These two functions are also the inverses of each other, when multiplication is taken as the tensor product with the trivial representation as the identity. This can be seen explicitly at any order in $t$ by observing that the tensor product $\left(\wedge^{p} R\right) \otimes\left(\vee^{q} R\right)$ generically contains exactly the plethysms described by the two different "hook" Young tableaux of $\mathfrak{s}(|R|)$ obtained by gluing together the column and the row describing the two factors. One thus has

$$
\mathscr{Z}_{R}^{F}(t) \otimes \mathscr{Z}_{R}^{B}(t)=1,
$$

It is then reasonable to use the formal notation

$$
\begin{aligned}
& \mathscr{Z}_{R}^{F}(t)=(1-t)^{R}, \\
& \mathscr{Z}_{R}^{B}(t)=(1-t)^{-R} .
\end{aligned}
$$

A fermion in $R$ can be seen as a boson in $-R$ and vice versa. It is important to understand the notation of eq. (3.5) as the shorthand it is, with eq. (3.3) being its defining expression.

All considerations of the refined partition functions may also be performed using characters, since they provide a ring homomorphism. Writing the character of the representation $R$ as $\chi(R)=\sum_{\mu \in \Lambda_{R}} e^{\mu}$, where $\Lambda_{R}$ is the set of weights for $R$, counting weights with multiplicities $m>1$ as $m$ "distinct" weights, we have

$$
\chi\left(\wedge^{k} R\right)=\sum_{\left\{\mu_{1}, \ldots, \mu_{k}\right\}} e^{\mu_{1}+\cdots+\mu_{k}},
$$

where the sum is over sets of distinct (in the sense above) weights in $\Lambda_{R}$. Thus,

$$
\chi_{R}^{F}(t) \equiv \chi\left(\mathscr{Z}_{R}^{F}(t)\right)=\sum_{k=1}^{|R|}(-t)^{k} \sum_{\left\{\mu_{1}, \ldots, \mu_{k}\right\}} e^{\mu_{1}+\cdots+\mu_{k}}=\prod_{\mu \in \Lambda_{R}}\left(1-t e^{\mu}\right),
$$

which of course is just the product of the characters for the individual fermions making up the representation $R$. It then follows that

$$
\chi_{R}^{B}(t) \equiv \chi\left(\mathscr{Z}_{R}^{B}(t)\right)=\left(\chi_{R}^{F}(t)\right)^{-1}=\prod_{\mu \in \Lambda_{R}}\left(1-t e^{\mu}\right)^{-1} .
$$

The character picture will be used for a proof of our result in section 5 . 
The examples above used for setting the notation are valid only for unconstrained variables (creation operators). We will use such refined partition functions to encode the spectrum of generators in the Borcherds superalgebras described in section 2.

Before going into the construction of partition functions for algebras and for constrained objects, we will consider two other situations, which will be of use later. The first is when a fermionic or bosonic variable is "maximally constrained", so that any bilinear vanishes. Then the partition function just contains a linear term:

$$
\mathscr{Z}(t)=1+\sigma R t
$$

(where again $\sigma= \pm 1$ for bosons and fermions, respectively). The second situation concerns variables of "indefinite statistics", meaning that both symmetric and antisymmetric products of $R$ occur (but with odd levels still labeled as bosonic or fermionic by a sign $\sigma$ ). Then the partition function is

$$
\mathscr{Z}(t)=\bigoplus_{p=0}^{\infty}(\sigma t)^{p} \otimes^{p} R=(1-\sigma R t)^{-1} .
$$

The observation that the partition functions (3.9) and (3.10) are each other's inverses for opposite choices of $\sigma$ is one, somewhat trivial, example of our main result which will be demonstrated in the following sections. In this case the algebra is freely generated by the representation at level one.

\section{BRST operator and coalgebra}

Consider the subalgebra $\mathscr{B}_{+}$of elements at positive levels of a $\mathbb{Z}$-graded Borcherds superalgebra $\mathscr{B}$, as defined in section 2 . In the generic case, the algebra will be infinite-dimensional, and contain elements at arbitrarily high levels. However, as we saw in section 2, all this information is contained in the covariant Serre relations

$$
\left.\left\{E_{M}, E_{N}\right\}\right|_{R_{2}^{\perp}}=0
$$

where $R_{2}{ }^{\perp}$ is the complement to $R_{2}$ in $\vee^{2} R_{1}$. At level two, we thus have generators $E_{M N}=\left\{E_{M}, E_{N}\right\}$ in $R_{2}$.

As announced in section 1, we will argue that all information about the representations occurring at each level can be obtained in an alternative way, which often provides a more direct answer, namely by considering a bosonic object $\lambda_{M}$ in $R_{1}$, subject to the constraint

$$
\left.\lambda^{2}\right|_{R_{2}}=0 .
$$

Notice that the object $\lambda_{M}$ has opposite statistics (bosonic) to $E_{M}$ (thinking of odd elements in a superalgebra as fermionic), and that its constraint is in the symmetric representation complementary to that of the Serre relations. The precise relation we will establish, and which is the main result of this paper, is:

The partition function of the universal enveloping algebra $U\left(\mathscr{B}_{+}\right)$is the inverse of the partition function for the constrained object $\lambda$, i.e.,

$$
\mathscr{Z}_{U\left(\mathscr{B}_{+}\right)}(t) \otimes \mathscr{Z}_{\lambda}(t)=1 .
$$


Since the partition functions used are completely refined, in the sense of section 3 , this provides complete information of the generators at each level of the Borcherds superalgebra $\mathscr{B}$. The refined partition function for $\lambda$, if $\lambda$ is seen as a complex object, can be seen as encoding holomorphic functions of $\lambda$.

The way we will argue for this equality in the present section is by identifying the action of the BRST operator for the (conjugated) constraint with the operation " $d$ " of the coalgebra $\mathscr{B}_{+}^{*}$. This will not constitute a full proof (which would require a consideration of cohomology of $\mathscr{B}_{+}^{*}$ ), but provides a clear picture of the correspondence. The proof, based on the denominator formula for Borcherds superalgebras, is given in section 5 .

Let us first consider the coalgebra, repeat some well known facts and set the notation. For simplicity, we do this for the case of an ordinary Lie algebra; the generalisation to graded brackets and Lie superalgebras is trivial. The coalgebra of a Lie algebra $\mathfrak{a}$ is defined on the vector space $\mathfrak{a}^{*}$ dual to $\mathfrak{a}$. It is equipped with a map $d: \mathfrak{a}^{*} \rightarrow \mathfrak{a}^{*} \wedge \mathfrak{a}^{*}$, which is dual to the Lie bracket $[\cdot, \cdot]$ in the sense that for any $A, B \in \mathfrak{a}$ and $X \in \mathfrak{a}^{*}$,

$$
\langle d X \mid A \wedge B\rangle=\langle X \mid[A, B]\rangle,
$$

where $\langle\cdot \mid \cdot\rangle$ is the canonical scalar product, naturally extended to tensor products. If $E_{a}$ and $E^{* a}$ are dual bases for $\mathfrak{a}$ and $\mathfrak{a}^{*}$, and $\left[E_{a}, E_{b}\right]=f_{a b}{ }^{c} E_{c}$, eq. (4.4) reads

$$
d E^{* a}=f_{b c}{ }^{a} E^{* b} \wedge E^{* c} .
$$

The action of $d$ is naturally extended to tensor products of elements by defining it to act as a derivation. The Jacobi identity is equivalent to the nilpotency, $d^{2}=0$, of $d$. The above can be generalised to a Lie superalgebra with the appropriate graded interpretation of wedge products, brackets and derivations.

We now specialise on the Borcherds superalgebras at hand. The first two levels of the coalgebra $\mathscr{B}_{+}^{*}$ read

$$
\begin{aligned}
d E^{* M} & =0, \\
d E^{* M N} & =\left.E^{* M} \vee E^{* N}\right|_{\bar{R}_{2}} .
\end{aligned}
$$

The Serre relations manifest themselves as the absence of generators in $\bar{R}_{2}{ }^{\perp}$ at level two. What is the procedure for the continued construction? Of course, knowledge of the algebra directly provides the full information of the coalgebra. But it is also possible to use eq. (4.6) as a starting point for recursively deriving the content at each level as well as the coproduct. One must then allow for the most general representation for $E^{*(3)}$ and the most general form of $d E^{*(3)} \sim E^{*(2)} \wedge E^{*(1)}$ consistent with $d^{2}=0$. A general Ansatz consists of letting $E^{* M N P}$ belong to a representation $R_{3} \subset R_{1} \otimes R_{2}$ and writing

$$
d E^{* M N P}=\left.E^{* M} \wedge E^{* N P}\right|_{\bar{R}_{3}} .
$$

The nilpotency of $d$ then determines the allowed representation $R_{3}$. For example, a totally symmetric representation is always excluded from $R_{3}$, since it will vanish due to the Jacobi identity. This procedure can then be continued to all levels, where $d E^{*(p)}$ will contain sums of terms $E^{*(q)} \wedge E^{*(p-q)}$ (wedge here denoting graded antisymmetrisation). 
The unique result of the procedure can be understood by the following argument, which also provides a conceptual idea behind the result stated in eq. (4.3). Everything starts from, and is generated from, the basic set of generators $E_{M}$ in the $\mathfrak{g}$-representation $\mathscr{B}_{1}$. Since they are odd elements of a superalgebra, they are normally thought of as fermionic. It is however useful to think of $E_{M}$ as not carrying a definite statistics. Indeed, considering the Serre relations (4.1), the only constraint on a bilinear in $E_{M}$ (including both symmetric and anti-symmetric parts) is that a certain representation $R_{2}{ }^{\perp}$ of the symmetric bilinear vanishes. The identification of the symmetric part in the complement $R_{2}$ with "new" generators $E_{M N}$ is in this sense optional. Without this identification, and corresponding identifications at higher levels, the universal algebra $U\left(\mathscr{B}_{+}\right)$can be constructed from the tensor algebra of $\mathscr{B}_{1}$ by factoring out the ideal generated by $\left.E_{M} \otimes E_{N}\right|_{R_{2} \perp}$. This provides a way of constructing an arbitrary element, not in the algebra $\mathscr{B}_{+}$, but in its universal enveloping algebra $U\left(\mathscr{B}_{+}\right)$, in terms of powers of $E_{M}$ only. The partition function of the universal enveloping algebra will be that of an object $E_{M}$ in $R_{1}$ of indefinite statistics (although the elements at odd levels are labelled as fermionic in the partition function, see section 3), modulo the ideal generated by the Serre relations. Seen this way, our main result can be phrased in the following way:

The partition function for a bosonic object $(\lambda)$ in $R_{1}$ subject to a bilinear constraint in $R_{2}$ is the inverse of the partition function for an object (the set of level-one generators in $\mathscr{B}$ ) with indefinite statistics, where odd powers are labeled as fermionic, subject to a bilinear constraint in $R_{2}{ }^{\perp}$.

This statement provides an interpolating generalisation for partitions of constrained objects of the ones made for unconstrained and maximally constrained ones in section 3. However, unlike in those limiting cases, the statistics here may not be switched, which we will comment on in section 6 .

Now, consider an object $\bar{\lambda}$ in $\bar{R}_{1}$, with the constraint $\left.\bar{\lambda}^{2}\right|_{\bar{R}_{2}}=0$. The constraint can be treated using a BRST formalism. For convenience, we change our notation and use $c^{M}$ instead of $\bar{\lambda}^{M}$. The first term in the BRST operator $Q$ is $Q_{(2)}=b_{M N} c^{M} c^{N}$, where $b_{M N}$ in $R_{2}$ is the ghost for the constraint. ${ }^{4}$ However, if the constraint happens to be reducible, there will be higher order ghosts compensating for the reducibility. Such reducibility will be captured by the introduction of a new $b c$ pair, and a term $Q_{(3)}=b_{M N P} c^{M N} c^{P}$ in $Q$. The representation of $b_{M N P}$ is everything that is allowed by $Q^{2}=0$. This should be continued, as long as the reducibility continues, i.e., as long as further such terms can be added. A generic term will be of the form $b_{(p+q)} c^{(p)} c^{(q)}$, where the ghosts are alternatingly fermionic and bosonic. From this trilinear form of the BRST operator it is immediately clear that its action on the $c$ ghosts defines the coalgebra of a Lie superalgebra. An infinite reducibility ${ }^{5}$ corresponds to an infinite-dimensional algebra.

\footnotetext{
${ }^{4}$ It would maybe be more conventional to use a notation where $c$ is the ghost multiplying the constraint, and $b$ its conjugate. Here, however, it turns out that all terms will be of the form $b c c$, which corresponds to the standard form of "algebra" ghost terms in a BRST operator.

${ }^{5}$ The concept of reducibility is not absolute, but may depend on the degree of covariance. Here, we always consider reducibility as expressed in terms of representations of the finite-dimensional Lie algebra $\mathfrak{g}$ (but should of course not be confused with the possible reducibility of the representations themselves).
} 
We now recognise the exact parallel between on one hand the construction of the coalgebra, given the Serre relations (and nothing more), and on the other hand the construction of the BRST operator. The difference is only a matter of notation. The cogenerators $E^{*(p)}$ correspond to the ghosts $c^{(p)}$, and the graded wedge products are automatically implied by the "wrong" statistics of the ghosts. The operator $d$ is the adjoint action (graded commutator) of $Q$, so that $d E^{*(p)} \leftrightarrow\left[Q, c^{(p)}\right\}$.

This means that if we calculate the partition function of $\lambda$ as a constrained object, which is obtained as the conjugate of the tensor product of the partition functions of all the ghosts,

$$
\mathscr{Z}_{\lambda}(t)=\bigotimes_{p=1}^{\infty}\left(1-t^{p}\right)^{(-1)^{p} R_{p}}
$$

it will coincide with the inverse partition function of the universal enveloping algebra $U\left(\mathscr{B}_{+}\right)$, which by definition is

$$
\mathscr{Z}_{U\left(\mathscr{B}_{+}\right)}(t)=\bigotimes_{p=1}^{\infty}\left(1-t^{p}\right)^{(-1)^{p+1} R_{p}},
$$

using the shorthand notation of (3.5). The inverse simply appears since the correspondence $E^{*(p)} \leftrightarrow c^{(p)}$ changes statistics.

The above argument does not provide a strict proof of eq. (4.3). The missing step is the proof that the BRST operator $Q \sim b c c$ correctly encodes the degrees of freedom of the constrained object, or, equivalently, that no other unwanted cohomology arises. We refrain from doing this, but we will present a different proof in section 5 .

Neither of the two above methods of finding the spectrum of generators has an advantage over the other, since we just demonstrated that they contain exactly the same calculational steps. However, knowing that the partition function is that of a constrained object $\lambda$ can often provide an alternative, more direct, and simpler way of obtaining the answer. Provided that we know from the constraint which representation $S_{p}$ appear at any power $\lambda^{p}$, the partition function is directly constructed as

$$
\mathscr{Z}_{\lambda}(t)=\bigoplus_{p=0}^{\infty} S_{p} t^{p}
$$

Expanding this partition function in a product form ${ }^{6}$ gives information about all the ghost representations, and thus about the generators of the algebra. This calculation becomes especially simple in cases where $S_{1}=R_{1}$ is an irreducible representation of some Lie algebra with highest weight $\lambda$, and $S_{2}=R_{2}{ }^{\perp}=\vee^{2} R_{1} \ominus R_{2}$ is the representation with highest weight $2 \lambda$. Then $S_{p}$ will have highest weight $p \lambda$. Indeed, the class of Borcherds superalgebras we consider all have this property, as will be shown in section 5 . We will give some examples of such situations in section 7, among which are pure spinors and their

\footnotetext{
${ }^{6}$ This is the method used by Berkovits and Nekrasov in ref. [23] to obtain detailed information on the partition functions of pure spinors.
} 
associated superalgebras. Although the representations $R_{p}$ are complicated, they can be calculated from the more readily available representations $S_{p}$ by inserting eqs. (4.9)-(4.10) into (4.3), which gives

$$
\bigotimes_{p=1}^{\infty}\left(1-t^{p}\right)^{(-1)^{p} R_{p}}=\bigoplus_{q=0}^{\infty} S_{q} t^{q}
$$

The explicit solution of this relation for the spectrum of the Borcherds superalgebra, i.e., the representations $R_{p}$ in terms of the known $S_{p}$, can be obtained by recursion, or by a Möbius inversion. By comparing the left and right hand sides for the first few powers of $t$ we get

$$
\begin{aligned}
& R_{1}=S_{1}, \\
& R_{2}=\vee^{2} R_{1} \ominus S_{2}, \\
& R_{3}=\left(R_{1} \otimes R_{2}\right) \ominus \vee^{3} R_{1} \oplus S_{3}, \\
& R_{4}=\left(\left(R_{1} \otimes R_{3}\right) \oplus \wedge^{2} R_{2}\right) \ominus\left(\vee^{2} R_{1} \otimes R_{2}\right) \oplus \vee^{4} R_{1} \ominus S_{4} .
\end{aligned}
$$

We will display some explicit examples of varying complexity in section 7 .

\section{A proof from denominator formulas}

This section will provide a proof of our main result (4.3), using the denominator formula for Borcherds superalgebras [31-34]. It is known for general Borcherds superalgebras but here we only need a simplified version given below, valid for the special cases of Borcherds superalgebras under consideration.

Let $\Phi$ be the root system of $\mathscr{B}$, and for any integer $p$, let $\Phi_{p}$ be the subset of $\Phi$ consisting of all roots $\beta=p \beta_{0}+\alpha$, where $\alpha$ is a linear combination of the real simple roots $\beta_{i}=\alpha_{i}$. Thus $\Phi_{0}$ is the root system of the subalgebra $\mathfrak{g}$, and $\mathscr{B}_{p}$ is the direct sum of all root spaces $\mathscr{B}_{\beta}$ such that $\beta \in \Phi_{p}$, and, if $p=0$, the Cartan subalgebra.

We will show that the eq. (4.11), with the lowest weights of the representations $S_{q}$ given by the Dynkin labels

$$
\lambda_{i}=q\left(\beta_{0}, \beta_{i}^{\vee}\right)=q \cdot 2 \frac{\left(\beta_{0}, \beta_{i}\right)}{\left(\beta_{i}, \beta_{i}\right)}=q B_{i 0},
$$

is equivalent to the denominator formula for $\mathscr{B}$ [31-34], which reads

$$
\frac{\prod_{\beta \in \Phi_{(0)}^{+}}\left(1-e^{-\beta}\right)^{\text {mult } \beta}}{\prod_{\beta \in \Phi_{(1)}^{+}}\left(1+e^{-\beta}\right)^{\text {mult } \beta}}=\sum_{w \in W} \sum_{q=0}^{\infty}(-1)^{|w|}(-1)^{q} e^{w\left(\rho-q \beta_{0}\right)-\rho} .
$$

Here $\Phi_{(0)}^{+}$and $\Phi_{(1)}^{+}$consist of all even and odd positive roots, respectively, $\rho$ is the Weyl vector of $\mathscr{B}$, defined by

$$
\left(\rho, \beta_{I}\right)=\frac{\left(\beta_{I}, \beta_{I}\right)}{2}
$$


and the Weyl group $W$ of $\mathscr{B}$ is generated by all fundamental Weyl reflections

$$
r_{i}: \beta \mapsto \beta-\left(\alpha_{i}^{\vee}, \beta\right)=\beta-2 \frac{\left(\alpha_{i}, \beta\right)}{\left(\alpha_{i}, \alpha_{i}\right)} .
$$

The length $|w|$ of an element $w$ in $W$ is the minimal number of fundamental Weyl reflections (not necessarily distinct), which, applied after each other, give $w$.

Note that the representations $S_{p}$ are given by the Dynkin labels $\lambda_{i}$ of their lowest weights, since we consider positive levels of $\mathscr{B}$. However, we are going to relate the denominator formula (5.2) for $\mathscr{B}$ to the character formula for $\mathfrak{g}$, which is usually expressed in terms of the highest weight of a representation. Therefore it is convenient to replace eq. (4.11) by the equivalent equation obtained by conjugating all representations,

$$
\bigotimes_{p=1}^{\infty}\left(1-t^{p}\right)^{(-1)^{p} \bar{R}_{p}}=\bigoplus_{q=0}^{\infty} \bar{S}_{q} t^{q}
$$

where now the highest weight of $\bar{S}_{q}$ is $-\lambda_{i}=-q B_{i 0}$. What we will actually show is that this eq. (5.5) is equivalent to the denominator formula $(5.2){ }^{7}$

Let $\tilde{\Lambda}_{0}$ be the element in the weight space of $\mathscr{B}$ such that $\left(\tilde{\Lambda}_{0}, \alpha_{i}\right)=0$ for all $i=1, \ldots, r$, and the componenent of $\tilde{\Lambda}_{0}$ corresponding to $\beta_{0}$ in the basis of simple roots is equal to one (this element exists uniquely since both $B_{I J}$ and $B_{i j}=a_{i j}$ are non-degenerate). Thus $\tilde{\Lambda}_{0}-\beta_{0}$ is an element in the weight space of $\mathfrak{g}$ (considered as a subspace of the weight space of $\mathscr{B})$. More generally, a root $\beta \in \Phi_{p}$ can be written $\beta=p \tilde{\Lambda}_{0}+\mu$, where $\mu=\beta-p \tilde{\Lambda}_{0}$ is an element in the weight space of $\mathfrak{g}$. We then get

$$
\begin{aligned}
\prod_{\beta \in \Phi_{p}}\left(1-e^{-\beta}\right)^{\text {mult } \beta} & =\prod_{\mu \in R_{p}}\left(1-e^{-p \tilde{\Lambda}_{0}} e^{-\mu}\right)^{\text {mult } \mu} \\
& =\sum_{k=0}^{\left|R_{p}\right|}(-1)^{k} \sum e^{-\left(\mu_{1}+\cdots+\mu_{k}\right)}\left(e^{-\tilde{\Lambda}_{0}}\right)^{k p}
\end{aligned}
$$

where the second sum goes over all sets of $k$ distinct weights $\mu_{1}, \ldots, \mu_{k}$ among the weights of $R_{p}$, counting (as in section 3 ) a weight with multiplicity $m$ as $m$ "distinct" weights. This sum can be obtained from the character for $\wedge^{k} R_{p}$ by inverting each term, which corresponds to conjugating the representation $R_{p}$. Thus

$$
\prod_{\beta \in \Phi_{p}}\left(1-e^{-\beta}\right)^{\operatorname{mult} \beta}=\sum_{k=0}^{\left|\bar{R}_{p}\right|}(-1)^{k} \chi\left(\wedge^{k} \bar{R}_{p}\right) s^{k p}
$$

where we have set $s=e^{-\tilde{\Lambda}_{0}}$. In the same way,

$$
\prod_{\beta \in \Phi_{p}}\left(1+e^{-\beta}\right)^{\text {mult } \beta}=\sum_{k=0}^{\left|\bar{R}_{p}\right|} \chi\left(\wedge^{k} \bar{R}_{p}\right) s^{k p}
$$

\footnotetext{
${ }^{7}$ Instead of considering positive levels in $\mathscr{B}$ and conjugating the representations we could of course also have considered negative levels and only highest weights from the beginning.
} 
and we know that the inverse of this is

$$
\prod_{\beta \in \Phi_{p}}\left(1+e^{-\beta}\right)^{- \text {mult } \beta}=\sum_{k=0}^{\infty}(-1)^{k} \chi\left(\bigvee^{k} \bar{R}_{p}\right) s^{k p} .
$$

Here the character of $\vee^{k} \bar{R}_{p}$ is given by the sum of all terms $e^{-\left(\mu_{1}+\cdots+\mu_{k}\right)}$, where $\mu_{1}, \ldots, \mu_{k}$ are weights of $R_{p}$, this time not necessarily distinct. Following the notation in section 3 , we write this as

$$
\prod_{\beta \in \Phi_{p}}\left(1 \pm e^{-\beta}\right)^{\mp \operatorname{mult} \beta}=\chi\left(\left(1 \pm s^{p}\right)^{\mp \bar{R}_{p}}\right)
$$

and the left hand side of eq. (5.2) becomes

$$
\frac{\prod_{\beta \in \Phi_{(0)}^{+}}\left(1-e^{-\beta}\right)^{\mathrm{mult} \beta}}{\prod_{\beta \in \Phi_{(1)}^{+}}\left(1+e^{-\beta}\right)^{\mathrm{mult} \beta}}=\prod_{\alpha \in \Phi_{0}^{+}}\left(1-e^{-\alpha}\right)^{\operatorname{mult} \alpha} \prod_{p=1}^{\infty} \chi\left(\left(1-(-1)^{p} s^{p}\right)^{(-1)^{p} \bar{R}_{p}}\right)
$$

where $\Phi_{0}^{+}$consists of the positive roots of $\mathfrak{g}$.

We now turn to the right hand side of the denominator formula,

$$
\sum_{w \in W} \sum_{q=0}^{\infty}(-1)^{|w|}(-1)^{q} e^{w\left(\rho-q \beta_{0}\right)-\rho} .
$$

Here $W$ is the Weyl group of $\mathscr{B}$, but since it is generated by the fundamental Weyl reflections corresponding to the real roots only, it coincides with the Weyl group of $\mathfrak{g}$. In order to use the character formula for $\mathfrak{g}$ we also need to replace the Weyl vector of $\mathscr{B}$ with the one of $\mathfrak{g}$, but this requires some more consideration. The Weyl vector $\rho=\rho_{\mathscr{B}}$ of $\mathscr{B}$ is defined as the element in the weight space of $\mathscr{B}$ satisfying

$$
\left(\rho_{\mathscr{B}}, \beta_{I}\right)=\frac{\left(\beta_{I}, \beta_{I}\right)}{2}
$$

whereas the Weyl vector $\rho_{\mathfrak{g}}$ of $\mathfrak{g}$ only has to satisfy

$$
\left(\rho_{\mathfrak{g}}, \alpha_{i}\right)=\frac{\left(\alpha_{i}, \alpha_{i}\right)}{2},
$$

but on the other hand it must have a zero component corresponding to $\beta_{0}$ in the basis of simple roots. Thus the Weyl vectors of $\mathscr{B}$ and $\mathfrak{g}$ are different (in general), but since their difference $\rho_{\mathscr{B}}-\rho_{\mathfrak{g}}$ is orthogonal to the real roots, $\left(\rho_{\mathscr{B}}-\rho_{\mathfrak{g}}, \alpha_{i}\right)=0$, it is invariant under the Weyl group, $w\left(\rho_{\mathscr{B}}-\rho_{\mathfrak{g}}\right)=\rho_{\mathscr{B}}-\rho_{\mathfrak{g}}$. We then get

$$
\begin{aligned}
w\left(\rho_{\mathscr{B}}-q \beta_{0}\right)-\rho_{\mathscr{B}} & =w\left(\rho_{\mathfrak{g}}+\left(\rho_{\mathscr{B}}-\rho_{\mathfrak{g}}\right)-q \beta_{0}\right)-\rho_{\mathfrak{g}}-\left(\rho_{\mathscr{B}}-\rho_{\mathfrak{g}}\right) \\
& =w\left(\rho_{\mathfrak{g}}-q \beta_{0}\right)-\rho_{g}+w\left(\rho_{\mathscr{B}}-\rho_{\mathfrak{g}}\right)-\left(\rho_{\mathscr{B}}-\rho_{\mathfrak{g}}\right) \\
& =w\left(\rho_{\mathfrak{g}}-q \beta_{0}\right)-\rho_{g}
\end{aligned}
$$


and we can indeed replace $\rho=\rho_{\mathscr{B}}$ by $\rho_{\mathfrak{g}}$ in eq. (5.12). To simplify the notation, we will henceforth write $\rho=\rho_{\mathfrak{g}}$. Furthermore, since also $\tilde{\Lambda}_{0}$ is orthogonal to the real roots, we have

$$
\begin{aligned}
w\left(\rho-q \beta_{0}\right)-\rho & =w\left(\rho-q \tilde{\Lambda}_{0}+q\left(\tilde{\Lambda}_{0}-\beta_{0}\right)\right)-\rho \\
& =w\left(\rho+q\left(\tilde{\Lambda}_{0}-\beta_{0}\right)\right)-\rho-q \tilde{\Lambda}_{0}
\end{aligned}
$$

and then

$$
\begin{aligned}
\sum_{w \in W} \sum_{q=0}^{\infty}(-1)^{|w|}(-1)^{q} e^{w\left(\rho-q \beta_{0}\right)-\rho} & =\sum_{w \in W} \sum_{q=0}^{\infty}(-1)^{|w|}(-1)^{q} e^{w\left(\rho+q\left(\tilde{\Lambda}_{0}-\beta_{0}\right)\right)-\rho-q \tilde{\Lambda}_{0}} \\
& =\sum_{w \in W} \sum_{q=0}^{\infty}(-1)^{|w|}(-1)^{q} e^{w\left(\rho+q\left(\tilde{\Lambda}_{0}-\beta_{0}\right)\right)-\rho} s^{q} .
\end{aligned}
$$

Equating eqs. (5.11) and (5.17) we get

$$
\prod_{p=1}^{\infty} \chi\left(\left(1-(-1)^{p} s^{p}\right)^{(-1)^{p} \bar{R}_{p}}\right)=\sum_{q=0}^{\infty} \frac{\sum_{w \in W}(-1)^{|w|} e^{w\left(\rho+q\left(\tilde{\Lambda}_{0}-\beta_{0}\right)\right)-\rho}}{\prod_{\beta \in \Phi_{0}^{+}}\left(1-e^{-\beta}\right)^{\text {mult } \beta}}(-1)^{q} s^{q},
$$

where we recognise

$$
\frac{\sum_{w \in W}(-1)^{|w|} e^{w\left(\rho+q\left(\tilde{\Lambda}_{0}-\beta_{0}\right)\right)-\rho}}{\prod_{\beta \in \Phi_{0}^{+}}\left(1-e^{-\beta}\right)^{\operatorname{mult} \beta}}
$$

as the character of the representation of $\mathfrak{g}$ with highest weight $q\left(\tilde{\Lambda}_{0}-\beta_{0}\right)$ given by the Dynkin labels

$$
\left(q\left(\tilde{\Lambda}_{0}-\beta_{0}\right), \beta_{i}^{\vee}\right)=-q\left(\beta_{0}, \beta_{i}^{\vee}\right)=-q \cdot 2 \frac{\left(\beta_{0}, \beta_{i}\right)}{\left(\beta_{i}, \beta_{i}\right)}=-q B_{i 0}=-\lambda_{i},
$$

and thus

$$
\prod_{p=1}^{\infty} \chi\left(\left(1-(-1)^{p} s^{p}\right)^{(-1)^{p} \bar{R}_{p}}\right)=\sum_{q=0}^{\infty} \chi\left(\bar{S}_{q}\right)(-1)^{q} s^{q} .
$$

Finally, substituting $s$ by $-t$ we arrive at the equation

$$
\prod_{p=1}^{\infty} \chi\left(\left(1-t^{p}\right)^{(-1)^{p} \bar{R}_{p}}\right)=\sum_{q=0}^{\infty} \chi\left(\bar{S}_{q}\right) t^{q}
$$

which is the character version of (and thus equivalent to) eq. (5.5).

\section{Why is the method not applicable to Lie algebras?}

Let us replace $\beta_{0}$ with an even simple root $\alpha_{0}$, which is real, $\left(\alpha_{0}, \alpha_{0}\right)>0$, but otherwise satisfies the same inner product relations as $\beta_{0}$, thus $\left(\alpha_{0}, \alpha_{i}\right)=\left(\beta_{0}, \alpha_{i}\right)$. The ChevalleySerre relations (2.4)-(2.5), with all superbrackets being ordinary antisymmetric brackets, and with the Cartan matrix $B_{I J}$ replaced by

$$
A_{I J}=\left(\alpha_{J}, \alpha_{I}^{\vee}\right)=2 \frac{\left(\alpha_{J}, \alpha_{I}\right)}{\left(\alpha_{I}, \alpha_{I}\right)},
$$


defines a Kac-Moody algebra $\mathscr{A}$. This corresponds to adding an ordinary (white) node to the Dynkin diagram of $\mathfrak{g}$ instead of a grey one, and in analogy with $\mathscr{B}$ the adjoint representation of $\mathscr{A}$ dcomposes into $\mathfrak{g}$-representations $R_{p}$. The representation $R_{1}$ is the same as in the case of $\mathscr{B}$, but $R_{2}$ is now a subrepresentation of $\wedge^{2} R_{1}$, the anti-symmetric tensor product of $R_{1}$ with itself. Its complement is the direct sum of representations with lowest weights given by the Dynkin labels $\lambda_{i}=A_{i j}+2 A_{i 0}$ for all $j$ such that $A_{0 j} \neq 0$.

One might imagine that the statement (4.3) would apply both for the Lie superalgebra $\mathscr{B}$ and the ordinary Lie algebra $\mathscr{A}$. This would potentially have made it possible to extract precise information about the generators to all levels for classes of infinite-dimensional (e.g. hyperbolic) Kac-Moody algebras. It turns out, however, that statistics can not just be changed. This is because the constrained object $\lambda$ then would be fermionic. Having bilinear (bosonic) constraints on fermionic variables is generically a strange situation, and leads to complicated structures, as we will explain.

Consider a fermionic $\lambda$ in a representation $R_{1}$ of $\mathfrak{g}$ with a bilinear constraint in $R_{2}$ of $\mathscr{A}$, thus complementary to some Serre relations in the anti-symmetric product $\wedge^{2} R_{1}$. An algebraic "solution" to the constraints (in the sense that one considers a power series in $\lambda$ modulo the constraint) will result in a polynomial partition function, where the highest term is of order lower than or equal to $\left|R_{1}\right|$. Its factorisation in ghost contributions is however infinite. This is because the ghosts, like the original variables, are fermionic, and so are the ghosts for reducibility. Instead of removing fermionic degrees of freedom, the ghosts add more fermions, corresponding to the removal of bosonic degrees of freedom (the constraint).

This somewhat pathological behaviour is in itself not an obstruction for the existence of a relation like eq. (4.3) - one might well imagine that a properly regularised sum with strictly positive terms yields a negative value (although it is a valid argument against an analogous construction when the Lie algebra is finite-dimensional). What makes things go wrong is the fact that a bilinear constraint on fermions inherently has some reducibility coming from the fermionic property of the variables. Whatever the bosonic constraint is, it is e.g. obvious that raising it to a sufficient power will give zero due to saturation of fermions. This has no counterpart in the bosonic situation, and will introduce ghosts in a BRST treatment which do not enter $Q$ in the " $b c c$ " form. Therefore, a correct BRST treatment can not be given an interpretation in terms of a Lie algebra. We have observed in a number of examples that a naive treatment of the Serre relations as complementary to a constraint gives agreement in the spectrum to a number of low levels, before the "saturation of fermions" becomes relevant. Whether there is a systematic way of consistently defining partitions for fermions that circumvents this and correctly encodes the Serre relations (and thereby the spectrum of the Lie algebra) is an open question. In any case it seems reasonable that the occurrence, in the superalgebra case, of the highest weights which are simply multiples of the defining one, has particularly simple structure without counterpart in the Lie algebra situation.

Turning to the actual proof of the main result for the Lie superalgebra $\mathscr{B}$ in section 5 , it is easy to identify the step where the argument fails for Lie algebras. The Weyl group of the extended algebra $\mathscr{A}$ is not identical to that of $\mathfrak{g}$, and the proof in its present form fails, although the denominator formula is known. 


\section{$7 \quad$ Examples}

We will give a number of examples that illustrate the connection between the constrained bosonic variable and the spectrum of generators in the superalgebra.

We use the notation $\mathscr{B}_{+}$for the subalgebra of generators at positive levels, although in some examples (the freely generated algebras) it is not a subalgebra of a Borcherds superalgebra of the precise type described in section 2 .

\subsection{The extreme cases}

Consider first a freely generated superalgebra. Then the Serre relations are empty, and elements of the universal enveloping algebra $U\left(\mathscr{B}_{+}\right)$are given by arbitrary tensor products of $R_{1}$. Thus $\mathscr{Z}_{U\left(\mathscr{B}_{+}\right)}$is given by eq. (3.10) with $\sigma=-1$, which is the inverse of the formal partition for a "maximally constrained" boson in $R_{1}$. This is one extreme case of the correspondence (in which the statistics can be interchanged). It appears if the additional simple root is not a null root, but has negative length squared.

The other extremal case is when the Serre relations fill the whole symmetric product $\vee^{2} R_{1}$, so that $\left\{E_{M}, E_{N}\right\}=0$. The superalgebra is then finite-dimensional with $E_{M}$ forming a basis for $\mathscr{B}_{+}=\mathscr{B}_{1}$. The partition function $\mathscr{Z}_{U\left(\mathscr{B}_{+}\right)}$is the partition function of fermions in $R_{1}$, which is the inverse of the partition function for an unconstrained boson. Also in this other special case the statistics can be interchanged.

Intermediary cases only work as a correspondence of the form (4.3) between superalgebras and constrained bosons, and these provide less trivial illustrations of our result.

\section{2 $D=8$ pure spinors and null vectors}

Pure spinors provide well known and extensively studied examples of constrained bosons. They lead to minimal spinor orbits under Spin groups, due to the fact that only a single irreducible representation appears in a spinor bilinear (the one whose highest weight is twice the one of a spinor), and by induction there is only one representation for each positive power of the spinor. We will give two examples of pure spinors, in this subsection and the next.

Let us first consider a pure spinor in $D=8$, where the constraint is particularly simple, $\lambda_{\alpha} \lambda_{\alpha}=0$. This is via triality equivalent to a null vector. The Dynkin diagram of the corresponding superalgebra is given below.

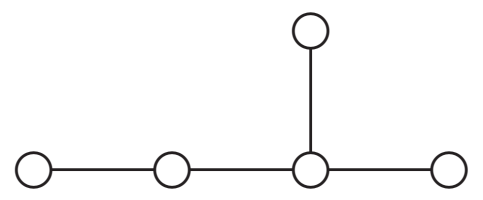

The analysis can equally well be performed for null vectors in general dimension $D$. The refined partition function for $\lambda$ reads

$$
\mathscr{Z}_{\lambda}(t)=\bigoplus_{p=0}^{\infty}(p 0 \ldots 0) t^{p},
$$


where Dynkin labels of highest weights have been used for the representations, and $(10 \ldots 0)$ denotes the vector representation. The representation $(p 0 \ldots 0)$ consists of symmetric and traceless multi-vectors. Its dimension is readily calculated to be

$$
\left(\begin{array}{c}
p+D-1 \\
p
\end{array}\right)-\left(\begin{array}{c}
p+D-3 \\
p-2
\end{array}\right)=\frac{(2 p+D-2)(p+D-3) !}{(D-2) ! p !},
$$

so the unrefined partition function (just counting dimensions) is

$$
Z_{\lambda}(t)=\sum_{p=0}^{\infty} \frac{(2 p+D-2)(p+D-3) !}{(D-2) ! p !} t^{p}=\frac{1+t}{(1-t)^{D-1}}=\frac{1-t^{2}}{(1-t)^{D}} .
$$

The same result is obtained by constructing the partition function from the ghosts. An (unconstrained) variable $\lambda$ contributes to $\mathscr{Z}$ with $(1-t)^{-(10 \ldots 0)}$, and the fermionic ghost for the constraint with $\left(1-t^{2}\right)^{(00 \ldots 0)}$. The constraint is irreducible, so there are no higher ghosts. The correspondence (4.3) tells us that the spectrum of the universal enveloping algebra $U\left(\mathscr{B}_{+}\right)$is given by

$$
\mathscr{Z}_{U\left(\mathscr{B}_{+}\right)}(t)=\left(\mathscr{Z}_{\lambda}(t)\right)^{-1}=(1-t)^{(10 \ldots 0)} \otimes\left(1-t^{2}\right)^{-(00 \ldots 0)},
$$

corresponding to a fermionic generator in $(10 \ldots 0)$ at level one and a bosonic one in $(00 \ldots 0)$ at level two. The superalgebra $\mathscr{B}$, which here comes with a 5 -grading, is finitedimensional, $\mathscr{B} \approx \mathfrak{o s p}(D \mid 2)$. A finite-dimensional superalgebra is obtained when the reducibility of the constraint on $\lambda$ is finite.

\section{3 $D=10$ pure spinors and supergravity forms}

Let us turn to the more interesting cases of infinite-dimensional superalgebras, which are related to the spectrum of forms in supergravity, and thereby also to the tensor hierarchies in gauged supergravity (see the discussion in section 1).

Pure spinors in $D=10$ are relevant for the off-shell superfield formulation of $D=10$ super-Yang-Mills theory (see e.g. refs. [35-38]). The partition function is described in some detail in ref. [23], and is given by

$$
\begin{aligned}
\mathscr{Z}_{\lambda}(t)= & \bigoplus_{p=0}^{\infty}(0000 k) t^{p} \\
= & {\left[(00000) \ominus(10000) t^{2} \oplus(00001) t^{3}\right.} \\
& \left.\ominus(00010) t^{5} \oplus(10000) t^{6} \ominus(00000) t^{8}\right] \otimes(1-t)^{-(00001)}
\end{aligned}
$$

or, just counting dimensions,

$$
Z_{\lambda}(t)=\frac{1-10 t^{2}+16 t^{3}-16 t^{5}+10 t^{6}-t^{8}}{(1-t)^{16}}=\frac{(1+t)\left(1+4 t+t^{2}\right)}{(1-t)^{11}} .
$$

The power 11 of the pole at $t=1$ signals 11 degrees of freedom in a pure spinor. The Dynkin diagram of the corresponding superalgebra is given below.

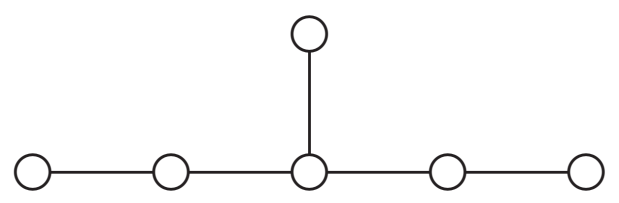


This algebra is infinite-dimensional. Still, we know that the spectrum is determined by $\mathscr{Z}_{U\left(\mathscr{B}_{+}\right)}=\mathscr{Z}_{\lambda}{ }^{-1}$. The generators at each level in $\mathscr{B}_{+}$are obtained by rewriting the partition function (7.5) on product form, which reflects the ghost structure corresponding to the infinite reducibility:

$$
\left(\mathscr{Z}_{\lambda}(t)\right)^{-1}=\bigotimes_{p=1}^{\infty}\left(1-t^{p}\right)^{(-1)^{p+1} R_{p}} .
$$

This can be done recursively as in eq. (4.12), with the following result for the first few representations:

$$
\begin{aligned}
& R_{1}=(00001)=\mathbf{1 6}, R_{2}=(10000)=\mathbf{1 0}, R_{3}=(00010)=\overline{\mathbf{1 6}}, \\
& R_{4}=(01000)=\mathbf{4 5}, R_{5}=(10010)=\overline{\mathbf{1 4 4}}, \\
& R_{6}=(11000) \oplus(00020) \oplus(10000)=\mathbf{3 2 0} \oplus \overline{\mathbf{1 2 6}} \oplus \mathbf{1 0}, \ldots
\end{aligned}
$$

For the dimensionalities $\left|R_{p}\right|$, an explicit Möbius inversion formula can be found [23].

The case of $D=10$ pure spinors is relevant to exceptional field theory with U-duality group $E_{5} \approx \operatorname{Spin}(5,5)$. Generally, the infinite ghost tower in exceptional field theory with U-duality group $E_{n}(n \leq 8)$ is identical to the infinite spectrum of superforms in $D$-dimensional maximal supergravity $(D=11-n)$, as was shown for low levels in ref. [22]. Our results here, combined with those in refs. [8-10], establish this correspondence for all levels. Here we have shown that the ghosts for a constrained object give rise to a Borcherds superalgebra by the action of the BRST operator, and in refs. [8-10] it was shown that the forms in the supergravity theory similarly give rise to a Borcherds superalgebra by their Bianchi identities. In the extended field theory the constraint is directly associated with the section condition, and leads to the same Borcherds superalgebras as the supersymmetry constraint on the supergravity side. Since the Borcherds superalgebras are the same, the sequences of representations are the same as well.

The (unrefined) partition functions corresponding to the constraint in the exceptional field theories were give in ref. [22]. As an example, the $E_{6}$ case gives a Borcherds superalgebra defined by a $\lambda$ belonging to a cône over the Cayley plane [39]. The partition function is

$$
\begin{aligned}
\mathscr{Z}_{\lambda}(t)= & \bigoplus_{k=0}^{\infty}(k 00000) t^{k} \\
= & {\left[(000000) \ominus(000001) t^{2} \oplus(010000) t^{3} \ominus(001000) t^{5}\right.} \\
& \oplus(100001) t^{6} \ominus(000002) t^{7} \ominus(200000) t^{8} \oplus(100001) t^{9} \\
& \left.\ominus(000010) t^{10} \oplus(010000) t^{12} \ominus(100000) t^{13} \oplus(000000) t^{15}\right] \\
& \otimes(1-t)^{-(100000)}
\end{aligned}
$$

and the spectrum of the Borcherds algebra is obtained recursively by rewriting $\mathscr{Z}_{\lambda}$ on product form.

With the same interpretation as gauge transformations and reducibility for generalised diffeomorphisms, our earlier example with null vectors, corresponding to a finitedimensional superalgebra, is relevant for double field theory with T-duality group $O(d, d)$. 


\subsection{Superalgebras and Lie algebras}

A curious observation, somewhat besides the main focus of this paper, is that the last Dynkin diagram of the previous subsection has the same form as the one for $E_{6}$, had the extra node been white instead of grey. Similarly, had the extra node in the superalgebra of subsection 7.2 been white, we would have had the Lie algebra for $\mathrm{SO}(10)$, or in the general case $\mathrm{SO}(D+2)$.

Polynomials of a pure spinor, the constrained object encoding the spectrum of the Borcherds algebra in question, indeed form an infinite-dimensional "singleton" representation of $E_{6}$, which can be constructed as follows. Consider generators of $E_{6} \supset \mathfrak{s o}(10) \oplus \mathfrak{u}(1)$. The adjoint splits as $\mathbf{7 8} \rightarrow \overline{\mathbf{1 6}}_{-1} \oplus(\mathbf{4 5} \oplus \mathbf{1})_{0} \oplus \mathbf{1 6}_{1}$. Call the spinorial generators $\lambda^{\alpha}$ and $\mu_{\alpha}$. With the conventions

$$
\left[J_{a b}, \lambda^{\alpha}\right]=\frac{1}{4}\left(\gamma_{a b} \lambda\right)^{\alpha}, \quad\left[Q, \lambda^{\alpha}\right]=\lambda^{\alpha}
$$

the only non-manifestly covariant non-vanishing commutator is

$$
\left[\mu_{\alpha}, \lambda^{\beta}\right]=\left(\gamma^{a b}\right)_{\alpha}^{\beta} J_{a b}+\frac{3}{2} \delta_{\alpha}^{\beta} Q
$$

The relative coefficient is fixed by demanding the Jacobi identity on the form

$$
\left[\left[\mu_{\alpha}, \lambda^{[\beta}\right], \lambda^{\gamma]}\right]=0
$$

Now, we start from an $\mathfrak{s o}(10)$-scalar "ground state" $|0\rangle$ annihilated by $\mu_{\alpha}$, and use $\lambda^{\alpha}$ as "creation operators", giving a Verma module of polynomials in $\lambda$. Let the ground state have charge $q, Q|0\rangle=q|0\rangle$. We want to adjust the value of $q$ so that $\left(\lambda \gamma^{a} \lambda\right)$ generates an $E_{6}$-invariant ideal. This happens if $\mu_{\alpha}\left(\lambda \gamma^{a} \lambda\right)|0\rangle=0$. A short calculation leads to

$$
\begin{aligned}
\mu_{\alpha}\left(\lambda \gamma^{a} \lambda\right)|0\rangle & =\frac{3}{2}(2 q+1)\left(\gamma^{a} \lambda\right)_{\alpha}|0\rangle+\frac{1}{4}\left(\gamma^{i j} \gamma^{a} \gamma_{i j} \lambda\right)_{\alpha}|0\rangle \\
& =\left(\frac{3}{2}(2 q+1)-\frac{27}{2}\right)\left(\gamma^{a} \lambda\right)_{\alpha}|0\rangle
\end{aligned}
$$

If $q=4$, this vanishes, and the ideal may be factored out without breaking $E_{6}$. This shows how the space of (holomorphic) polynomials in a pure spinor forms an infinite-dimensional lowest-weight representation of $E_{6}$. It may be called a singleton representation, since it only consists of a "leading trajectory" of Spin(10) representations with highest weights $(0000 k)$ at each $\mathrm{U}(1)$ charge $4+k$. The lowering operator $\mu_{\alpha}$ can be identified with the gauge invariant (in the sense that it respects the ideal) derivative with respect to a pure spinor constructed in ref. [40].

In the same way a singleton representation of $\mathrm{SO}(D+2)[41]$ is obtained by starting from a ground state of a certain charge. We use the conventions

$$
\begin{aligned}
{\left[J_{a b}, \lambda_{c}\right] } & =2 \lambda_{[a} \eta_{b] c}, \quad\left[Q, \lambda_{a}\right]=\lambda_{a} \\
{\left[\mu_{a}, \lambda_{b}\right] } & =J_{a b}-\eta_{a b} Q .
\end{aligned}
$$


The ideal generated by $\lambda^{2}$ can be factored out for vacuum charge $q=\frac{D-2}{2}$, leading to a singleton representation.

At least in these particular cases, the constrained object, i.e., the pure spinor or null vector, has a direct relation both to a Borcherds superalgebra and to a (finite-dimensional) Lie algebra. Both algebras are obtained by adding a node, grey and white, respectively, in the same position to the Dynkin diagram of a semi-simple Lie algebra, but the rôle of the pure spinor is quite different in the two cases. The phenomenon is certainly more general, but is in its present form limited to situations where the $\lambda$ 's commute, and thus are the only generators at positive level in the Lie algebra. It holds e.g. for the constrained objects $\lambda$ occurring in relation to tensor hierarchies of $E_{n} \times \mathbb{R}^{+}$with $n \leq 6$, and the Borcherds superalgebras obtained by extending $E_{n}$ by an odd null root. The corresponding Lie algebra, having polynomials of $\lambda$ as a singleton representation is $E_{n+1} \supset E_{n} \times \mathbb{R}^{+}$, which is a 3 -grading.

\section{Acknowledgments}

MC would like to thank Sergio Benvenuti and Amihay Hanany for pointing out parallels with the work of refs. [42, 43], where similar partition functions occur e.g. in relation to moduli spaces of instantons. JP would like to thank Thomas Edlund, Alex Feingold, Sebastian Guttenberg, Axel Kleinschmidt and Arne Meurman for discussions. The work of JP is supported by NSF grant PHY-1214344.

Open Access. This article is distributed under the terms of the Creative Commons Attribution License (CC-BY 4.0), which permits any use, distribution and reproduction in any medium, provided the original author(s) and source are credited.

\section{References}

[1] E. Cremmer, B. Julia, H. Lü and C.N. Pope, Dualization of dualities. 2. Twisted self-duality of doubled fields and superdualities, Nucl. Phys. B 535 (1998) 242 [hep-th/9806106] [INSPIRE].

[2] P. Henry-Labordere, B. Julia and L. Paulot, Borcherds symmetries in M-theory, JHEP 04 (2002) 049 [hep-th/0203070] [inSPIRE].

[3] M. Henneaux, B.L. Julia and J. Levie, E_11, Borcherds algebras and maximal supergravity, JHEP 04 (2012) 078 [arXiv: 1007.5241] [InSPIRE].

[4] E.A. Bergshoeff, M. de Roo, S.F. Kerstan and F. Riccioni, IIB supergravity revisited, JHEP 08 (2005) 098 [hep-th/0506013] [INSPIRE].

[5] E.A. Bergshoeff, M. de Roo, S.F. Kerstan, T. Ortín and F. Riccioni, IIA ten-forms and the gauge algebras of maximal supergravity theories, JHEP 07 (2006) 018 [hep-th/0602280] [INSPIRE].

[6] E.A. Bergshoeff, J. Hartong, P.S. Howe, T. Ortín and F. Riccioni, IIA/IIB supergravity and ten-forms, JHEP 05 (2010) 061 [arXiv: 1004.1348] [INSPIRE].

[7] J. Greitz and P.S. Howe, Maximal supergravity in three dimensions: supergeometry and differential forms, JHEP 07 (2011) 071 [arXiv:1103.2730] [INSPIRE]. 
[8] J. Greitz and P.S. Howe, Maximal supergravity in D =10: forms, Borcherds algebras and superspace cohomology, JHEP 08 (2011) 146 [arXiv:1103.5053] [INSPIRE].

[9] J. Greitz and P.S. Howe, Half-maximal supergravity in three dimensions: supergeometry, differential forms and algebraic structure, JHEP 06 (2012) 177 [arXiv:1203.5585] [INSPIRE].

[10] P. Howe and J. Palmkvist, Forms and algebras in (half-)maximal supergravity theories, JHEP 05 (2015) 032 [arXiv: 1503.00015] [INSPIRE].

[11] J. Palmkvist, Tensor hierarchies, Borcherds algebras and E11, JHEP 02 (2012) 066 [arXiv: 1110.4892] [INSPIRE].

[12] J. Palmkvist, Borcherds and Kac-Moody extensions of simple finite-dimensional Lie algebras, JHEP 06 (2012) 003 [arXiv: 1203.5107] [INSPIRE].

[13] F. Riccioni and P.C. West, The $E_{11}$ origin of all maximal supergravities, JHEP 07 (2007) 063 [arXiv:0705.0752] [INSPIRE].

[14] E.A. Bergshoeff, I. De Baetselier and T.A. Nutma, $E_{11}$ and the embedding tensor, JHEP 09 (2007) 047 [arXiv: 0705.1304] [INSPIRE].

[15] E.A. Bergshoeff, J. Gomis, T.A. Nutma and D. Roest, Kac-Moody spectrum of (half-)maximal supergravities, JHEP 02 (2008) 069 [arXiv:0711.2035] [INSPIRE].

[16] B. de Wit, H. Samtleben and M. Trigiante, Gauging maximal supergravities, Fortsch. Phys. 52 (2004) 489 [hep-th/0311225] [INSPIRE].

[17] B. de Wit and H. Samtleben, Gauged maximal supergravities and hierarchies of non-Abelian vector-tensor systems, Fortsch. Phys. 53 (2005) 442 [hep-th/0501243] [INSPIRE].

[18] B. de Wit, H. Nicolai and H. Samtleben, Gauged supergravities, tensor hierarchies and M-theory, JHEP 02 (2008) 044 [arXiv:0801.1294] [INSPIRE].

[19] B. de Wit and H. Samtleben, The end of the p-form hierarchy, JHEP 08 (2008) 015 [arXiv: 0805.4767] [INSPIRE].

[20] J. Palmkvist, The tensor hierarchy algebra, J. Math. Phys. 55 (2014) 011701 [arXiv: 1305.0018] [INSPIRE].

[21] J. Greitz, P. Howe and J. Palmkvist, The tensor hierarchy simplified, Class. Quant. Grav. 31 (2014) 087001 [arXiv:1308.4972] [INSPIRE].

[22] D.S. Berman, M. Cederwall, A. Kleinschmidt and D.C. Thompson, The gauge structure of generalised diffeomorphisms, JHEP 01 (2013) 064 [arXiv:1208.5884] [INSPIRE].

[23] N. Berkovits and N. Nekrasov, The character of pure spinors, Lett. Math. Phys. 74 (2005) 75 [hep-th/0503075] [INSPIRE].

[24] M. Cederwall, J. Edlund and A. Karlsson, Exceptional geometry and tensor fields, JHEP 07 (2013) 028 [arXiv: 1302.6736] [INSPIRE].

[25] O. Hohm and H. Samtleben, U-duality covariant gravity, JHEP 09 (2013) 080 [arXiv: 1307.0509] [INSPIRE].

[26] O. Hohm and H. Samtleben, Exceptional field theory I: $E_{6(6)}$ covariant form of M-theory and type IIB, Phys. Rev. D 89 (2014) 066016 [arXiv:1312.0614] [InSPIRE].

[27] O. Hohm and H. Samtleben, Exceptional field theory. II. $E_{7(7)}$, Phys. Rev. D 89 (2014) 066017 [arXiv:1312.4542] [INSPIRE]. 
[28] O. Hohm and H. Samtleben, Exceptional field theory. III. E $E_{8(8)}$, Phys. Rev. D 90 (2014) 066002 [arXiv: 1406.3348] [INSPIRE].

[29] V.G. Kac, Lie superalgebras, Adv. Math. 26 (1977) 8 [inSPIRE].

[30] V.G. Kac, Infinite dimensional Lie algebras, Cambridge University Press, Camrbidge U.K. (1990).

[31] M. Wakimoto, Infinite-dimensional Lie algebras, American Mathematical Society, U.S.A. (2001).

[32] U. Ray, Automorphic forms and Lie superalgebras, Springer, Germany (2006).

[33] U. Ray, A character formula for generalized Kac-Moody superalgebras, J. Algebra 177 (1995) 154.

[34] M. Miyamoto, A generalization of Borcherds algebra and denominator formula, J. Algebra 180 (1996) 631.

[35] M. Cederwall, B.E.W. Nilsson and D. Tsimpis, The structure of maximally supersymmetric Yang-Mills theory: constraining higher order corrections, JHEP 06 (2001) 034 [hep-th/0102009] [INSPIRE].

[36] N. Berkovits, Covariant quantization of the superparticle using pure spinors, JHEP 09 (2001) 016 [hep-th/0105050] [INSPIRE].

[37] M. Cederwall, B.E.W. Nilsson and D. Tsimpis, Spinorial cohomology and maximally supersymmetric theories, JHEP 02 (2002) 009 [hep-th/0110069] [INSPIRE].

[38] M. Cederwall, Pure spinor superfields - An overview, Springer Proc. Phys. 153 (2014) 61 [arXiv: 1307.1762] [INSPIRE].

[39] M. Cederwall, Jordan algebra dynamics, Phys. Lett. B 210 (1988) 169 [InSPIRE].

[40] M. Cederwall, Operators on pure spinor spaces, AIP Conf. Proc. 1243 (2010) 51 [inSPIRE].

[41] P.A.M. Dirac, A remarkable representation of the $3+2$ de Sitter group, J. Math. Phys. 4 (1963) 901 [inSPIRE].

[42] S. Benvenuti, A. Hanany and N. Mekareeya, The Hilbert series of the one instanton moduli space, JHEP 06 (2010) 100 [arXiv: 1005.3026] [INSPIRE].

[43] A. Hanany and R. Kalveks, Highest weight generating functions for Hilbert series, JHEP 10 (2014) 152 [arXiv:1408.4690] [INSPIRE]. 